\title{
A Novel Fuzzy Diffusion Approach for Improving Energy Efficiency in Wireless Sensor Networks
}

\author{
Faraneh Zarafshan, Member, IACSIT, Abbas Karimi, Member, IACSIT and S. A. R. Al-Haddad
}

\begin{abstract}
Directed Diffusion is a Data Centric routing protocol. In data centric protocols data is named based on the attribute-value pairs. Directed diffusion protocol includes flooding the interested data, establishing direction paths by using gradients set-up and selecting one or more direction paths to reinforce the data stream. The main difficulty of Directed Diffusion is flooding in which there is some communication overhead with some nodes which are naturally unable to coordinate in monitoring the interested event. We use a fuzzy logic controller to reduce the communication overheads during flooding and routing the data stream from the source(s) to a sink node. The fuzzy logic controller evaluates the potentiality of intermediate nodes to coordinate in mission, based on each node's traffic load, energy residual and size of data which can be maintained. The simulation results show that new Fuzzy diffusion approach produces at most $56.5 \%$ and in average $\mathbf{2 8 . 8 1 \%}$ successful results and has improved the total energy residual at least $19.38 \%$ and in average $1888.87 \%$ in comparison with directed diffusion protocol.
\end{abstract}

Index Terms-Wireless sensor networks, fuzzy logic, directed diffusion, energy efficiency.

\section{INTRODUCTION}

A Wireless sensor networks (WSN) contain multitude tiny nodes in which various sensors, actuators, processing and communication units exist. These nodes interact highly with physical environment providing continual unattended monitoring of natural or man-made phenomena and less accessible regions such as poisonous or chemical areas in industries, aircraft interiors and early warning of natural disasters [1]-[2]. These numerous sensing nodes distributed in an area can coordinate with each other and constitute a distributed network to achieve a larger sensing task [3].

Wireless sensor nodes are highly restricted by energy level of nodes. Although the recent advances in MEMS and VLSI technologies can lead to building very small and energy efficient sensor nodes enabling to do processing and computation, the demand on decreasing the energy consumption in nodes is still crucial even in some situations where the renewable energy sources (e.g. solar panel) are provided.

Besides hardware optimizations, energy efficient routing methods act a significant role in improving the routing

Manuscript received May 25, 2012; revised July 27, 2012.

F. Zarafshan and A. Karimi are with the Department of Computer Engineering, Faculty of Engineering, Arak Brach, Islamic Azad University, Arak, Iran (e-mail: fzarafshan@gmail.com; akarimi@iau-arak.com).

S. A. R. Al-Haddad is with Department of Computer and Communication Systems Engineering, Faculty of Engineering, Putra University, Serdang, Malaysia (e-mail: SAR@eng.upm.edu.my). energy efficiency.

From a point of view, routing methods are categorized into Data Centric (DC) and Address Centric (DC) [4]. DC protocols are significantly different from AC protocols where each node has a unique ID, the routing is end-to-end and each source independently send data along the shortest path to sink based on the routes the queries took [4]. However, in DC protocols there is no globally unique ID. The nodes are capacitated to handle the content of the data and perform some data aggregation on the sensed data which is passed via neighbor nodes.

Data aggregation (the combination of data from different sources [4]) dedicates many advantages to Data Centric Models. Of the advantages is eliminating the redundancy in intermediate nodes which often occurs in hop-by-hop routing methods. If two or more similar data packets enter an intermediate node, it can merge the same data and update their required fields to eliminate data redundancies. Since, sink often deals with the high traffic of processing data, in this way DC routing will not impose further loads to the sink for processing iterative data, besides less networks communications. Contrarily, in AC routing, the in-network processing is not allowed and the sink is responsible for all processing functions. So the iterative data may be sent to sink via different sources [4].

Directed Diffusion [2] is a Data Centric and application-aware routing model in which the communications are based on named data and the routing is short-range hop-by-hop. The data is named using an attribute-value pair [2]. This approach is request-based, so that the sink propagates a request- called as interest containing named data to some or all sensor nodes. Each node which has received the interest and decides to cooperate in the task announces its cooperation by setting-up a gradient to the neighbor which has already sent the interest. This process continues until a node which witnesses the event receives the interest and starts sending back the data with low rate to the originator of the interest, possibly along multiple gradient paths which are already established. Then among the multiple paths, one or more are reinforced for high rate data stream by the sink, until one or more sensors along the main path fail or sink finds other suitable sources. If so, a substituting path might be chosen via negative reinforcement.

The main difficulty with Directed diffusion is flooding for route discovery which wastes a considerable amount of energy especially in the intermediate nodes that are not able to coordinate. Although by some mechanisms we can send interests to a limited number of nodes, the only choice is flooding the interest all over the network, if there is no idea about the likely region of the source. 
In data centric approaches such as directed diffusion sensor nodes may deal with different sizes of data, so that a node can support a large capacity of data e.g. video types, while another can maintain medium or small size e.g. audio or image data. In this case, routing the unsuitable size of data to a node wastes the node's energy level due to imposed extra communications and processing functions and even leads to node breaking down or deadlock in a part of sensor network.

In this paper, we present a novel fuzzy approach to determine the potentiality of the nodes along routing path. By avoiding the nodes with low potentiality, we decrease the energy consumption of directed diffusion routing protocol with which the traffic load and size of interested data will be taken into account, besides the energy considerations in each node.

This paper is organized as following. In Section 2, the related works are presented. The assumptions, system description and fuzzy logic controller are introduced in Section 3. The simulation and test bed are presented in Section 4. The final section is conclusion and future works.

\section{RELATED WORKS}

The Directed Diffusion paradigm was introduced and analyzed experimentally in [5] for utilizing the communication and computation capability of sensor nodes to perform a distributed sensing of environment. Although they showed that directed diffusion is potentially energy efficient, it doesn't resolve the needs for reduction in its energy consumption.

So far, several researches have been conducted on different aspects of directed diffusion for reducing the energy consumption. A data propagation method which uses a threshold value to restrict the rate of data transmission during propagation and consequently improves energy consumption is introduced in [6]. In [7] an extension to directed diffusion known as Multi-path directed diffusion (MDD) is introduced which balances the traffic loads among sensor nodes and decreases the energy consumption due to spread overhead between multiple nodes. A path reinforcement technique is introduced in [8] that outperforms the directed diffusion in energy efficiency, energy balance over WSNs, and network lifetime.

Although the idea of using fuzzy logic to improve routing algorithms [6], [9] - [11] is not new, in Directed diffusion it is not considered much. In [1] a fuzzy diffusion approach for prolonging the life time of distributed sensor networks has been presented which is based upon the energy level and the traffic intensity of each node while a fuzzy multi-path routing scheme is presented in [12] taking the battery power level and the kind of data received from the neighbors into account.

\section{FUZZY DIFFUSION}

Owing to advances in VLSI and MEMS technologies, it is possible to perform computation and processing inside very tiny sensor nodes which are the basis of Data Centric routing algorithms including Directed Diffusion. They also make it possible to implement Fuzzy Logic Controller (FLC) with high functional speed and low power consumption [13]-[15].
The architecture of fuzzy logic controller is perceived in Fig. 1 .

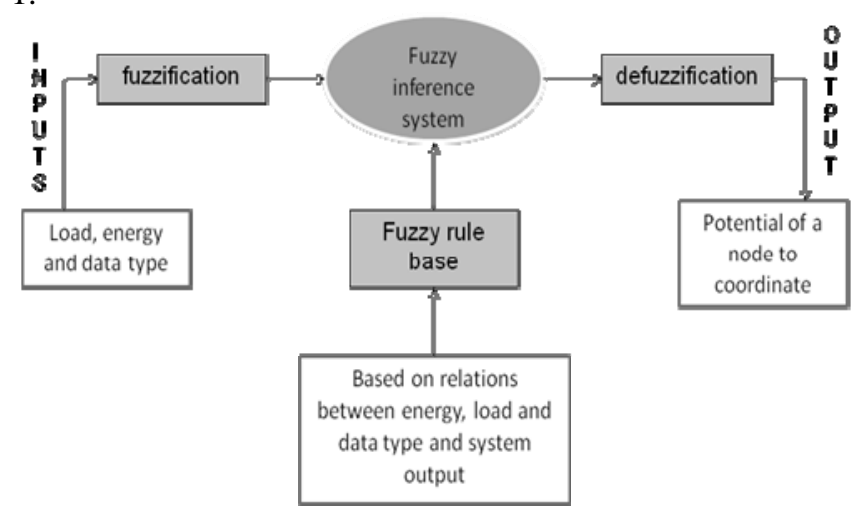

Fig. 1. The architecture of fuzzy logic controller

We use a fuzzy Logic Controller inside each sensor node with which the dynamic routing is possible. The fuzzy logic controller is responsible for converting crisp values for size of data, load status and energy residual in each node to fuzzy values and makes decision by using fuzzified inputs and defined rule base to provide a criterion by which we are able to measure the potential of the node for cooperating in the task. During flooding in fuzzy diffusion, the nodes with low potential can decide not to set-up the gradients and consequently in data propagation they likely will not cooperate in routing. Then no extra overhead is imposed on weak nodes and their energy is saved for network vital operations.

When an interest is received by a node, node can decide based on its potentiality which is calculated by using the values of traffic load, size of data and energy residual of a node and its neighbors. These parameters can be attained simply by following criteria.

Energy Level (EL) can be calculated by using the node's energy level and the minimum and maximum energy of the neighbors (Emin and Emax). The advantage of using the EL is that it examines a node's energy with respect to its neighbor status:

$$
E L=\left(E_{\text {node }}-E_{\min }\right) /\left(E_{\max }-E_{\min }\right)
$$

Since the communications between neighbors to get energy information are exchanged through piggy backing and are maintained in node's cache, their effect on network performance is insignificant.

In FLC, Trapezoidal function for mapping EL to low, moderate, high and full energy levels is considered as shown in Fig. 2.

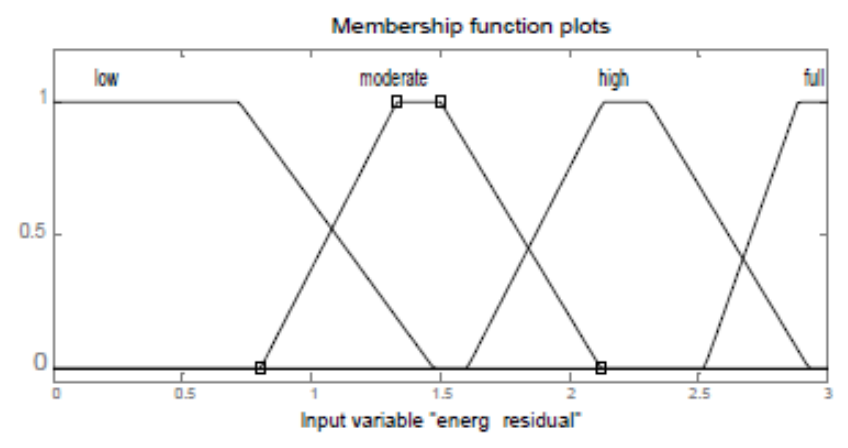

Fig. 2. Membership function for energy level 
Traffic Load (TL) is a measure to evaluate the traffic of each node considering the number of processing task in the queue and the maximum size of queue:

$$
\begin{aligned}
& T L=\text { Traffic in the node's queue/Maximum queue size } \\
& \text { of the node }
\end{aligned}
$$

Fuzzy interface system maps TL into 5 linguistic variables including very light, light, moderate, heavy and very heavy as presented in Fig. 3.

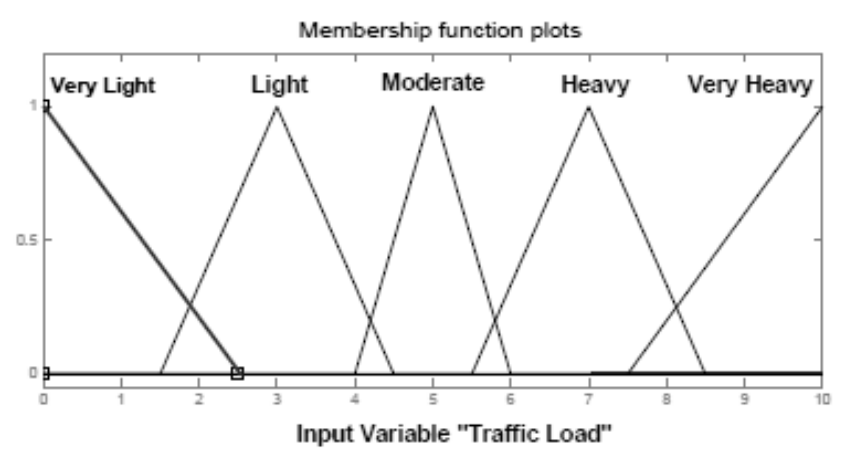

Fig. 3. Member function for Traffic Load

The size of data (SD) can be determined from the requested interest. In some situations it can be assumed as a way to estimate type of data, for instance if the requested size is small, it can be assumed to be an image; otherwise it is supposed to be an audio (if size is medium) or video (if size is large). Membership function for SD is illustrated in Fig. 4. This parameter is mapped by FLC to small, moderate, and large.

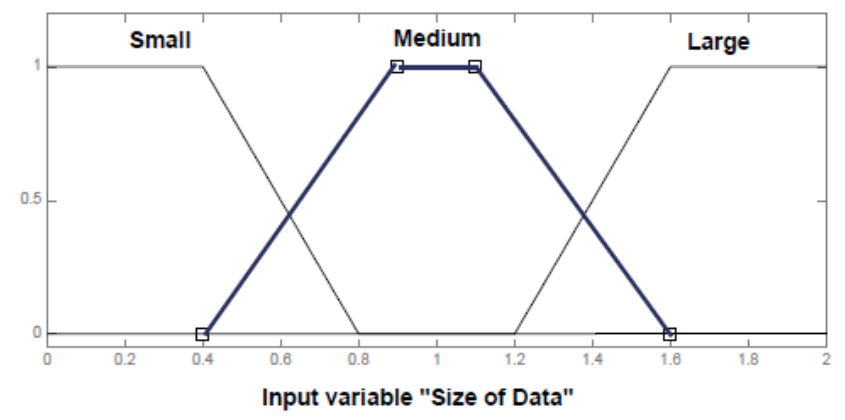

Fig. 4. Membership function for size of data

It is worthy to mention that the considered values in FLC for fuzzy inputs are arbitrary and can be modified by the user for specific applications. SD is assumed at most $2 \mathrm{Mb}$.

Our proposed fuzzy interface system is in Mamdani's form and relates linguistic inputs to fuzzy output based on defined fuzzy rule base. An example of a fuzzy rule is:

\section{If traffic load is very light and \\ Energy level is full and \\ Size of data is small \\ Then potentiality is very high.}

The output of the fuzzy interface system is the potentiality of a node in form of linguistic expressions which is modified to crisp values by a defuzzifier in which Centroid method has been used.

\section{EXPERIMENTAL RESULTS}

We simulate our fuzzy routing approach in MATLAB for a wireless sensor network comprising $N$ sensor nodes distributed in a $100 \mathrm{~m} \times 100 \mathrm{~m}$ area by Uniform distribution. The sink node is located at the center of wireless sensor network and it has no limitations on the energy, traffic load and size of data requested. Energy and traffic load of other nodes are initially supposed to be 3 joules and 5 packets in the queue, respectively. In each routing cycle a node is randomly chosen as the source node. When a sensor node sends a packet to a candidate neighbor, the energy and load of sender and the energy of receiver are decreased, whereas the load of receiver is increased. The amount of energy decrement is determined by Heinzelman's formula [16].

The size of interested data is determined by random function and distributed via requested interest during the routing operation. Each sensor node who has received the interest, calculate its own potentiality to cooperate in routing task. If the node does not have enough potentiality, it assign zero to its set gradient flag (reset the flag), otherwise it forwards the interest to its neighbors until the owner of data received the interest. Then, the owner sets its flag and starts routing process by backward method. In this phase, the source of data (owner of data) will choose a node with maximum potentiality among the neighbors who have set their flags. Since the protocol is hop-by-hop, selected neighbor acts as a new source for its neighbors. The routing process continues similarly, until the data receives to the sink. We consider a constant value as the packet time out so that if the packet is not received during the pre-determined time, it will be removed from the network and "packet lost" is reported.

The platform of our simulated wireless sensor network is illustrated in Fig. 5.

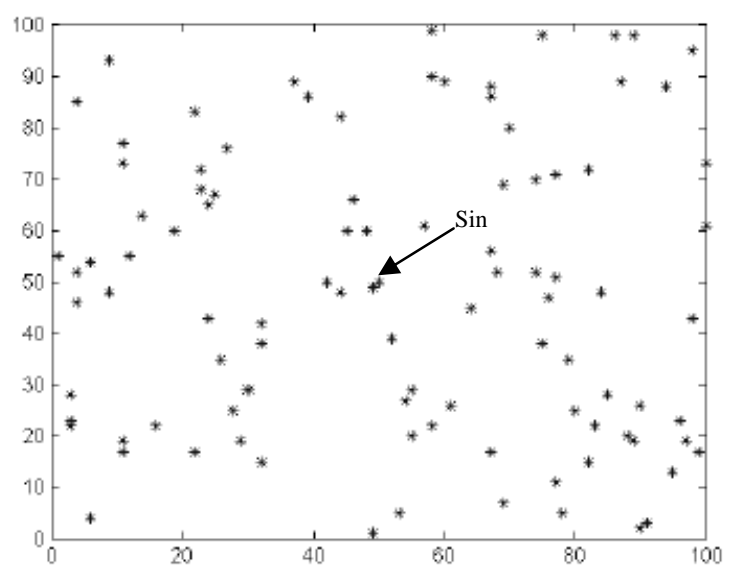

Fig. 5. Platform of simulated wireless sensor networks

Directed Diffusion algorithm has been also simulated with similar assumptions.

The success events of our fuzzy diffusion and directed diffusion protocols are compared in Fig. 6. The $\mathrm{x}$ axis shows the number of transmitted packets and $y$ axis refers to the number of success events. Our simulations show that Fuzzy diffusion produces at most $56.5 \%$ and in average $28.81 \%$ successful results in comparison with directed diffusion. 


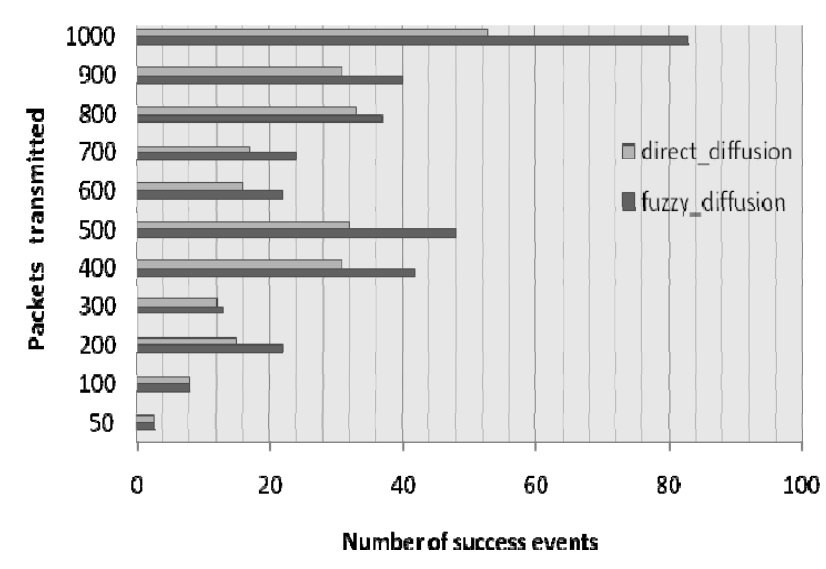

Fig. 6. Success events of fuzzy diffusion and directed diffusion

The average energy residual in wireless sensor nodes at the end of routing in terms of number of transmitted packets is illustrated in Fig. 7.

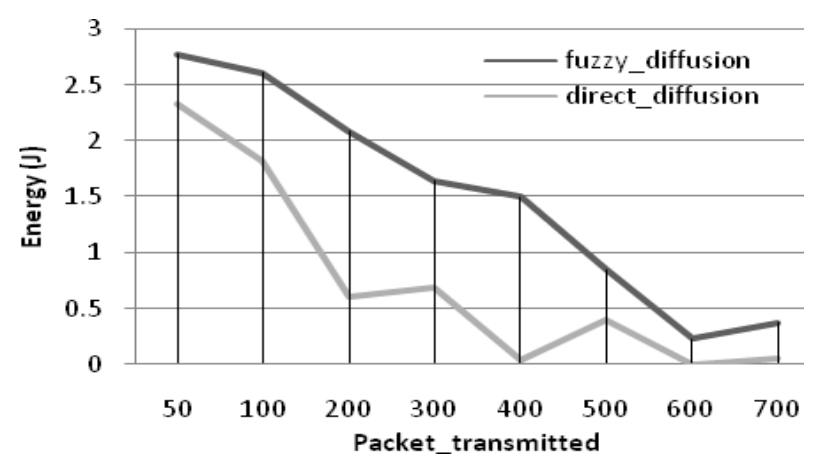

Fig. 7. Average energy residual in nodes

As it is clear the new fuzzy diffusion routing has more energy efficiency than directed diffusion approach and has improved the total energy residual at least $19.38 \%$ and in average $1888.87 \%$ in comparison with directed diffusion protocol.

\section{CONCLUSION AND FUTURE WORKS}

In this paper, a fuzzy diffusion approach based on directed diffusion routing protocol has been introduced. We assume a built-in fuzzy logic controller in each sensor node which determines the potential of a node for cooperation in a sensing task by using the current energy level and traffic load, besides the ability of a node to transmit specific size data which is determined by the propagated interest. The simulation results of new fuzzy approach and directed diffusion demonstrated that the new approach maintains more amounts of the energy residual in sensor nodes which means saving more energy in sensor nodes for future actions and prolonging life time of wireless sensor network. New approach also does more successful routing actions during the life time of network. Hence, the new fuzzy diffusion approach is energy efficient and improves the traditional directed diffusion routing protocol.

Furthermore, our fuzzy routing idea is basically capable to use with other routing protocols to enhance their energy efficiency.
In future works, the results of this research will be used and extended to other types of data centric routing protocols in wireless sensor networks.

\section{REFERENCES}

[1] M. Balakrishnan, and E. Johnson, "Fuzzy diffusion for distributed sensor networks", In Proc. IEEE Military Communications Conf. (MILCOM 2005), Atlantic City, NJ, 2005, vol 5, pp. 3023-3028.

[2] C. Intanagonwiwat, R. Govindan, D.Estrin, J. Heidemann, and F. Silva "Directed diffusion for wireless sensor networking”, IEEE/ACM Trans. on Networking, vol. 11, pp. 2-16, 2003.

[3] G. J. Pottie and W. J. Kaiser, "Wireless Integrated Network Sensors", Communications of the ACM, vol. 43, pp. 551-8, 2000.

[4] B. Krishnamachari, D. Estrin, and S. Wicker, "Modeling data-centric routing in wireless sensor networks", In Proc. of IEEE INFOCOM, New York, NY, 2002, vol. 2, no. 4.

[5] C. Intanagonwiwat, R. Govindan, and D. Estrin, "Directed Diffusion: A Scalable and Robust Communication Paradigm for Sensor Networks", In Proc. of the 6th annual int. conf. on Mobile Computing and Networking, 2000, Boston, Massachusetts, United States, pp. 56-67.

[6] S. H. Chi, T. H. Cho, "Fuzzy Logic Based Propagation Limiting Method for Message Routing in Wireless Sensor Networks", Computational Science and Its Applications - ICCSA, vol. 3983/2006, pp.58-67, ed Berlin / Heidelberg: Springer, 2006.

[7] A. N. Eghbali, M. Dehghan, "Load-Balancing Using Multi-path Directed Diffusion in Wireless Sensor Networks”, Mobile Ad-Hoc and Sensor Networks, vol. 4864/2007, pp.44-55, ed Berlin / Heidelberg: Springer, 2007.

[8] S. Zhao, Y. Fengqi, Z. Baohua, "An Energy Efficient Directed Diffusion Routing Protocol”, In Proc. the Int. Conf. on Computational Intelligence and Security (CIS 2007), Harbin, Heilongjiang, China, 2007, pp.1067-1072.

[9] L. Wang, S. Chen, L. Xiaojian, “A Multiple-objective Fuzzy Decision Making Based Information-aware Routing Protocol for Wireless Sensor Networks", In Proc. the Int. Conf. on Wireless Communications, Networking and Mobile Computing. WiCOM 2006, Wuhan, 2006, pp. 1-4.

[10] G. Li, Y. Cao, X. Gao, and J. Tang, "Energy balance routing protocol for wireless sensor networks based on fuzzy next-hop selection”, Wuhan University Journal of Natural Sciences, vol. 14, pp. 148-152, 2009.

[11] T. Haider and M. Yusuf, “A Fuzzy Approach to Energy Optimized Routing for Wireless Sensor Networks”, The International Arab Journal of Information Technology, vol. 6 , pp. 179-186, 2009.

[12] T. Srinivasan, R. Chandrasekar, and V. Vijaykumar, "A fuzzy, energy-efficient scheme for data centric multipath routing in wireless sensor networks", Presented at the IFIP International Conference on Wireless and Optical Communications Network, Bangalore, 2006.

[13] B. Singh, R. Goyal, R. Kumar, and R. P. P. Singh, "Design and VLSI implementation of Fuzzy Logic Controller”, International Journal of Computer and Network Security (IJCNS), vol. 1, no. 3, pp. 77-8, 2009.

[14] T. Daud, A. Stoica, T. Thomas, W. Li, and J.A. Fabunmi, "ELIPS: Toward a sensor fusion processor on a chip”, SPIE proceedings series, 1999, vol. 3719, pp. 209-219.

[15] A. D. Stefano and C. Giaconia, “An FPGA-Based Adaptive Fuzzy Coprocessor”, Computational Intelligence and Bioinspired Systems, vol. 3512/2005, pp. 590-597, ed Berlin / Heidelberg: Springer, 2005.

[16] W. R. Heinzelman, A. Chandrakasan, and H. Balakrishnan, "Energy-Efficient Communication Protocol for Wireless Microsensor Networks", In the Proceedings of the 33rd Hawaii International Conference on System Science, 2000, vol. 8, pp. 8020.

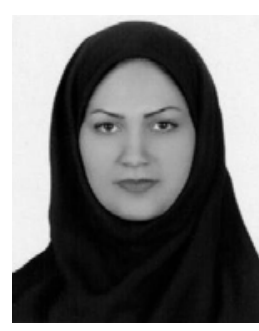

Faraneh Zarafshan is currently a $\mathrm{PhD}$ candidate in computer systems engineering with UPM and she also works as a researcher with Arak Branch, Islamic Azad University. She was the leader of multiple research projects, the author of three textbooks, and multiple journal and conference papers. She is a senior member of IACSIT, and a member of SDIWC. Her research interests include sensor network, real-time systems, and fault-tolerant systems. 


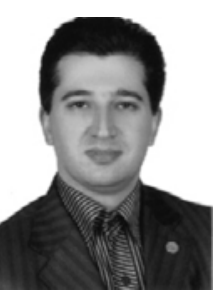

Abbas Karimi received his $\mathrm{PhD}$ degree in computer system engineering from Putra University, Malaysia (UPM). He works as a lecturer and a faculty member with the department of computer engineering, Arak Branch, Islamic Azad University. He was the leader of multiple research projects, and the author of three textbooks, multiple journal and conference papers. $\mathrm{He}$ is a senior member of IACSIT (International Association of Computer Science and Information Technology) and reviewer of multiple journals, such as JEST, IAJIT (the International Arab Journal of Information Technology), Engineering Letters, Entertainment ACM(Association of Computer Machinery), IJACSA (International Journal of Advanced Computer Science and Applications), and IJCSIS (International Journal of Computer Science and Information Security). His research interests include load balancing algorithms, real-time, distributed, and parallel and fault-tolerant systems.
S. A. R. Al-Haddad received his B.S. degree from Technology University, Malaysia (UTM), in 1997. He received his M.S. degree from UPM, in 2000. He received his Ph.D. degree from National University, Malaysia (UKM), in 2010. He has served as a lecturer with the Department of Computer Engineering and Communications System, Faculty of Engineering, UPM, since 11 May, 2000. His research interests include speech / voice / sound recognition, spoken/natural language understanding, dialog modeling, natural language generator, text to speech computer telephony integration, and office automation based fax and artificial intelligence. 